\title{
Development of Heart Rate Circadian Rhythm in Chickens
}

\author{
K. Moriya $^{a}$, R. Akiyama ${ }^{b}$, E.M. Dzialowski ${ }^{c}$,W.W. Burggren ${ }^{c}$ and H. Tazawa ${ }^{b}$ \\ ${ }^{a}$ Department of Electrical and Electronics Engineering, Hakodate National College of Technology, Hakodate 042-8501, \\ Japan \\ ${ }^{\mathrm{b}}$ Department of Electrical and Electronics Engineering, Muroran Institute of Technology, Muroran 050-8585, Japan \\ ${ }^{\mathrm{c}}$ Department of Biological Sciences, University of North Texas, Denton, Texas, USA
}

\begin{abstract}
In chick embryos, various instantaneous heart rate (IHR) fluctuations (e.g. HR variability, HR irregularities) have been found and developmental patterns of mean heart rate (MHR) have been elucidated. IHR changes have also measured in newly hatched and young chickens in order to investigate the developmental patterns of MHR and any potential diurnal HR rhythmicity such as a circadian rhythm. The present report reviews measurements of embryonic and hatchling HR fluctuations and examine development of HR circadian rhythms during the late stages of incubation and after hatching. Embryonic $\mathrm{HR}$ was continuously measured during the last half period of incubation. After hatching, continuous measurements of HR were made for two weeks at the longest in eight chickens ranging in age from 0 to 47 days. During the embryonic development, circadian rhythm of HR was found to be absent until hatching. After hatching, distinct circadian rhythm of HR appeared with increasing daily MHR from day 0 to day 7. During the second week of post-hatch life, the daily scope of MHR (diurnal maximum-nocturnal minimum) reached a maximum level ranging from 200 to $300 \mathrm{bpm}$ with constant baseline of nocturnal MHR. In young chicks, the nocturnal HR baseline was the same as that during the fortnight of posthatch life and daily scope of MHR decreased to $100-150 \mathrm{bpm}$.
\end{abstract}

Keywords: circadian rhythm, developmental pattern, chick hatchlings, mean heart rate, heart rate fluctuation, continuous measurement

\section{INTRODUCTION}

Daily rhythmicities are well known in the chicken, and includes rhythms in daily egg laying, calling at dawn, and daily changes in physiological functions such as metabolic rate (Berman et al., 1978) and brain temperature (Aschoff et al., 1976). These daily fluctuations are thought to be controlled by the daily cycle in hormones, such as melatonin (Herichová et al., 2001; Zeman et al., 2001) and the suprachiasmatic nucleus, pineal gland, and eye in the chicken (Yoshimura et al., 2001). Many of the proposed mechanisms that function as part of the internal clock have been shown to become entrained on the light:dark cycle and develop in the chicken prior to hatching (Zeman et al., 1999; Herichová et al., 2001; Yoshimura et al., 2001; Willbold et al., 2002; Okabayashi et al., 2003; Haque et al., 2003).
Heart rate (HR) of adult chickens also exhibits a circadian rhythm (Cain et al., 1970, 1974; Shimada and Koide, 1978; Tazawa et al., 1992) that appears to be entrained by light conditions (i.e. light intensity with light: dark cycle). During the last decade, continuous measurements of instantaneous heart rate (IHR) for long periods extending over a few days and weeks have been made in avian embryos and hatchlings. Accordingly, daily changes in HR can be shown as time-series patterns that visually indicate the absence or presence of circadian rhythm and are subject to regression analysis. The circadian rhythm was found to be absent in the continuous measurements of HR in chick embryos, but to be present after hatching (Moriya et al., 2000; Tazawa et al., 2002). This report reviews continuous measurements of HR in embryos and hatchlings and examines the circadian rhythm of the HR.

*To whom correspondence should be addressed: e-mail: moriya@hakodate-ct.ac.jp 


\section{REVIEW OF METHODOLOGY}

\subsection{Continuous measurements of heart rate in embryos and hatchlings}

Fertile eggs of broiler chickens that were brought from a local hatchery to a laboratory in Muroran Institute of Technology were used for experiments. Eggs were incubated at $38^{\circ} \mathrm{C}$ and about $60 \%$ relative humidity in a forced-draught incubator with automatic turning every 3 hours. HR measurement in embryos developing inside an eggshell variously used acoustocardiogaphy with a condenser microphone (Rahn et al., 1990; Wang et al., 1990; Akiyama et al., 1997), and electrocardiography using wire electrodes. The former determined continuous recordings of HR during the last half of incubation up to about initiation of internal pipping. On around days $11-12$ of incubation, the condenser microphone was attached hermetically on the eggshell and measured an acoustocardiogram (ACG) (Akiyama et al., 1997; Akiyama et al., 1999). IHR in beats $/ \mathrm{min}^{-1}(\mathrm{bpm})$ was calculated from an inverse of time interval between the two adjacent peaks of the ACG. The non-invasive determination of HR was continued during the last half of incubation (Akiyama et al., 1999). When embryonic activities augmented and breathing movements occurred towards the end of incubation on days 18-19, the microphone began to record these signals as well as the ACG. The ACG signal was very minute compared with signals attributable to somatic and breathing activities and so it became inadequate for continuous measurement of HR during the last stages of incubation. Alternatively, electrocardiography was used to supplement continuous HR recordings from a period prior to pipping up to hatching (Moriya et al., 2000). Silver wire electrodes were implanted in the egg through pinholes opened in the eggshell so that three locations for electrodes formed a triangle to enclose the late embryo. Electrocardiogram (ECG) was continuously measured and IHR was determined from the adjacent two $\mathrm{R}$ waves.

For hatched chicks, continuous determination of HR was made by measurement of ECG (Moriya et al., 1999). When a chick hatched, it was removed from the incubator for the connection of electrodes, which was made within a 30 minute period. Three flexible $\mathrm{Ag} / \mathrm{AgCl}$ electrodes were attached to the skin under the wings and abdomen. Electrode wires were fixed above the chick's back with an adhesive tape so that it could move freely and take food and water ad libitum. The chick was placed in a metal-meshed experimental box kept at $35^{\circ} \mathrm{C}$ with natural light conditions of about 12-13 hours light and about $11-12$ hours dark. The time of light-phase was counted based on the sunrisesunset time announced by a local meteorological observatory. Weather and amount of insolation announced by the observatory were also recorded. ECG was continuously measured for the two weeks at the longest. In case of measurements that were begun after one week post-hatch, the hatched chicks were reared at about $30^{\circ} \mathrm{C}$ with natural light conditions. Then, the chick was attached the electrodes and placed in the experimental box, and HR measurement was started after the chick acclimated to experimental temperature of $35^{\circ} \mathrm{C}$ for $1-2$ hours.

\subsection{Regression analysis}

Daily changes in MHR were analysed using following cosine function:

$$
\begin{aligned}
\text { MHR }= & -|A+B \cdot x| \cdot \cos (2 \pi x / T)+C \cdot x \\
& +H R_{\text {int }}(\mathrm{bpm})
\end{aligned}
$$

where $x$ (in day post-hatch) is age, $A$ (in bpm) is the initial amplitude of the circadian rhythm, $B$ (in bpm per day) is daily change in amplitude, $T$ (in days) is the period of the circadian rhythm, $C$ (in bpm per day) is the daily change in MHR and $\mathrm{HR}_{\text {int }}$ (in bpm) is the yaxis intercept (Moriya et al., 2002). Since the HR level at midnight is the lowest, amplitude of the cosine function has negative amplitude on the assumption that the phase parameter is the zero.

\section{DAILY CHANGES IN MHR AND HR CIRCADIAN RHYTHM}

An example of continuous recording of MHR during the last half of incubation in a chick embryo is shown in Figure 1 (Akiyama et al., 1999; Tazawa et al., 2002). On day 12 of incubation, the microphone was glued hermetically on the eggshell and ACG was measured continuously until it was disturbed by hatching and breathing activities after the last half of day 18. MHR over the 5-minute period (5-minute MHR) was calculated from the continuous measurement of ACG signals and was plotted in the upper panel by an individual single point. MHR in this embryo increased on days $14-15$ and then decreased, 


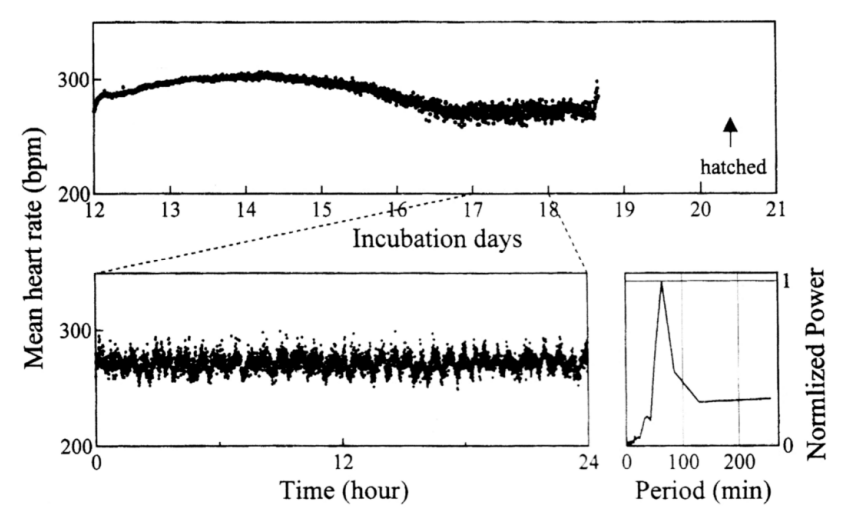

Fig. 1 The continuous measurement of MHR during the last half incubation in a pre-pipped chick embryo by acoustcardiography. The top panel shows the continuous recording of MHR averaged over 5-minute from day 12 of incubation. The bottom panel presents the 24-hour recording of MHR averaged over 20-second during days 17-18 extracted from the upper panel (left panel) and its power spectrum made by the fast Fourier transform (right panel). Modified from Akiyama et al. (1999).

widening the HR baseline on days 17-18. Apparently, no diurnal (or daily) HR rhythm was observed visually. Instead, ultradian rhythm of MHR occurred in the wide range HR baseline as shown in the left lower panel that was the 24-h recording of 20-s MHR from day 17. Power spectrum analysis made by the fast Fourier transform for the left recording of 20-s MHR indicates the oscillatory period of 65 minutes. This embryo hatched at the beginning of day 21 and the recording of HR by means of ACG was discontinued for about 2 days from the beginning of day 19. Alternatively, for late embryos undergoing hatching and breathing activities, the continuous recording of $\mathrm{HR}$ is made by measurement of ECG.

A developmental pattern of MHR as determined from an ECG before, during and after hatching period from day 18 of incubation to day 2 post-hatch is shown in Figure 2 (Moriya et al., 2000). HR baseline was wide and decreases towards internal pipping (IP). The wide ranging baseline for HR was attributed to the ultradian HR oscillation that was already obtained by ACG measurement. The ultradian HR oscillation continued up to the middle of gradual increase in HR baseline that occurred during the last half of day 19 with the subsequent fall originated by external pipping (EP) at the beginning of day 20. After the first about 12-hours of the
EP period in this embryo, HR increased distinctly to the high and wide range baseline. The distinct increase in HR baseline was due to relatively long-lasting cyclic small accelerations, and the following high and wide range HR baseline was attributed to alternate occurrences of short-term repeated large accelerations and irregular intermittent large accelerations (Tazawa et al., 1999). Besides these accelerations, HR oscillation with a period of $1-1.5$ seconds (i.e. respiratory sinus arrhythmia) occurred during the EP period. Although the HR fluctuations occurred on various periods ranging from several beats to about 90 min during the last week of incubation with particular emphasis during pipping period, circadian rhythm of HR was found to be absent in embryos. In general, the HR baseline that increased prior to hatching decreased transiently while the plumage was wet after hatching, but increased again to the pre-hatching high level that was maintained for several hours even during the night time (Moriya et al., 2000). For the individual animal whose record is shown in Figure 2, HR baseline decreased for several hours soon after hatching with a subsequent increase. HR baseline remained at a high value half that of the daytime phase on day 1 , then dropped during following night phase and subsequently rose during the daytime phase. A distinct circadian rhythm of HR began to occur after hatching.

Figure 3 shows a typical example of daily changes in MHR (i.e. developmental pattern) during the first week

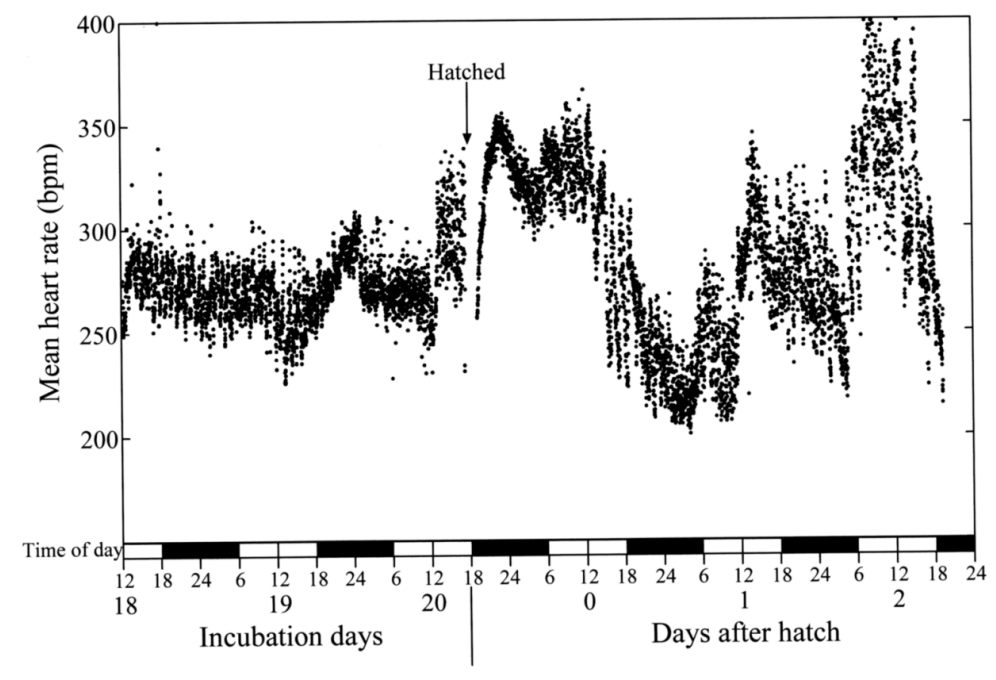

Fig. 2 Developmental pattern of MHR in a chick embryo during the last stages of incubation. The horizontal axis indicates the time of incubation in days, and the black boxes indicate periods of night-time phase from 18:00 to 06:00. Each black dot indicates the MHR over a 1-minute period. Symbol EP shows initiation of external pipping. Modified from Moriya et al. (2000). 


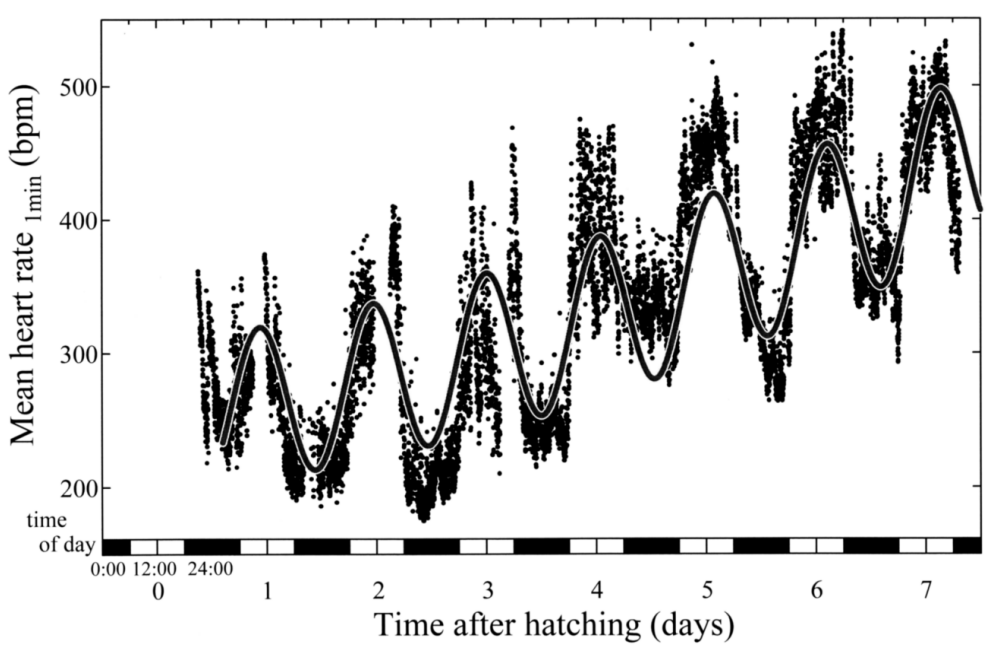

Fig. 3 The continuous measurement of MHR (dot) and its regression curve (solid line) described by

$M H R=-|53+1.1 \cdot x| \cdot \cos (2 \pi x / 1.06)+30 \cdot x+204 \quad($ bpm $)(r=0.88)$ during the first week of post hatch life. Each point indicates the MHR over a 1minute period. The horizontal axis indicates the time of day in days after hatch, and the black boxes indicate periods of night time phase from 18:00 to 06:00. The hatching day was designated as day 0. Modified from Moriya et al. (1999).

of post-hatching life obtained from a newly hatched chick (Moriya et al., 1999). The hatchling represented in Figure 3 had an increased HR baseline when measurement was begun at night. Then, the HR baseline decreased with time elapsed during the night and increased during the day, showing distinct circadian rhythm. The HR differences between nocturnal minimum and diurnal maximum increased from about $100 \mathrm{bpm}$ on day 0 to about $250 \mathrm{bpm}$ on day 6 . MHR averaged over an individual day (daily MHR) increased from about $250 \mathrm{bpm}$ on day 0 to about $450 \mathrm{bpm}$ on day 7 . In five other chicks, circadian rhythm of HR appeared on day 0 onward and daily MHR increased with days during the first week of post-hatching life. In one instance, circadian rhythm of HR was observed on day 3 onward with daily increase in daily MHR.

The developmental pattern of MHR during the first week post-hatch in seven chicks was approximated by the following equation:

$$
\begin{aligned}
\text { MHR }= & -|34+5 \cdot x| \cdot \cos (2 \pi x / 0.98) \\
& +15 \cdot x+277 \quad(\text { bpm })
\end{aligned}
$$

where the correlation coefficient $(r)$ was 0.73 and individual parameters were averaged. The mean period of circadian rhythm was 0.98 day (about 23.5 hours). The daily change in the amplitude of circadian rhythm and the daily change of daily MHR were $5 \mathrm{bpm}$ and $15 \mathrm{bpm}$, respectively, indicating that MHR increased up to day 7 with augmentation of amplitude. In newly hatched chicks, it is reported that the light conditions (i.e. light-dark period and light intensity) and food supply (i.e. energy metabolism) effect the circadian rhythm of HR (Shimada and Koide, 1978; Nichelmann et al., 1999).

The diurnal MHR during the second week of post-hatching life was higher than the nocturnal HR (Figure 4) and the differences extended over $200 \mathrm{bpm}$ except day 9 when the scope of diurnal and nocturnal MHR's was about $100 \mathrm{bpm}$. On day 9 post-hatching, which has a small scope of MHR changes, weather was cloudy and rainy and the amount of insolation was only $4.8 \mathrm{MJ} / \mathrm{m}^{-2}$. In other days, weather was cloudless and insolation was $23.2 \mathrm{MJ} / \mathrm{m}^{-2}$, for example, on day 13 . This result agrees with the hypothesis that light intensity influences the circadian rhythm of chick HR (Shimada and Koide, 1978).

The developmental patterns of MHR during the second week of post-hatch in three chicks are approximated by the following equation:

$$
\begin{aligned}
\text { MHR }= & -|72+0.3 \cdot x| \cdot \cos (2 \pi x / 0.99)-2 \cdot x \\
& +383 \text { (bpm) }
\end{aligned}
$$

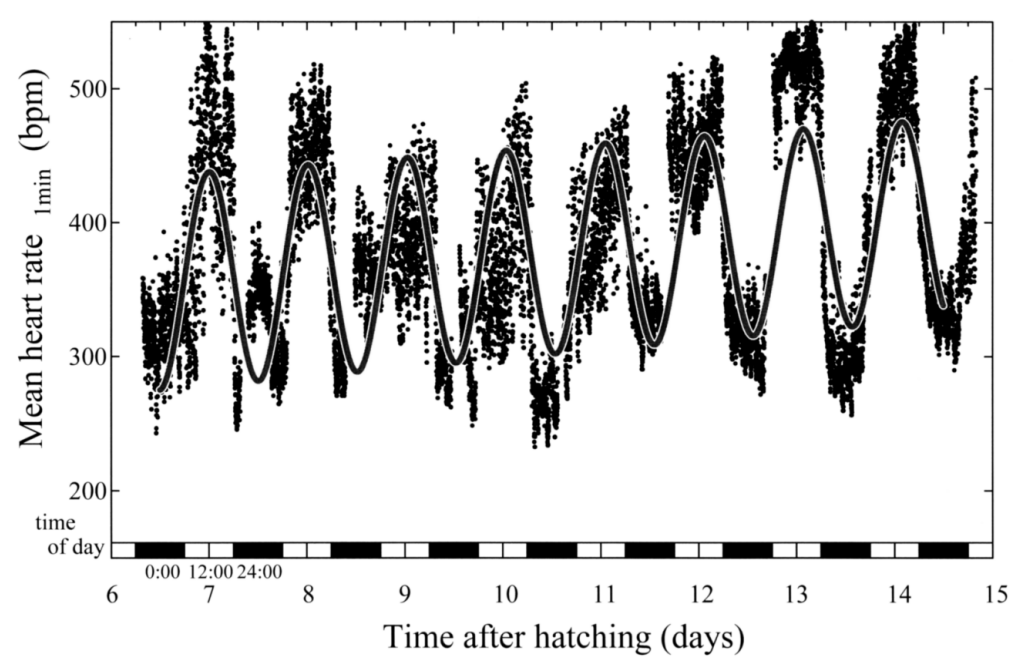

Fig. 4 An example of the MHR changes and its fitted function represented by $M H R=-|76-0.9 \cdot x| \cdot \cos (2 \pi x / 1.00)+6 \cdot x+313 \quad($ bpm $)(r=0.74)$ during the second week of post hatch life. Each dot indicates the MHR over a 1minute period. This is a different chicken from that in Fig. 3. 
Where $r=0.79$. The mean period of circadian rhythm during the second week was 0.99 day (about 23.8 hours). Both the daily changes in amplitude and daily MHR became small or negative, indicating that the scope of diurnal and nocturnal HR and daily MHR reached almost maximum level.

The developmental pattern of MHR in a chicken during the period from day 40 to 47 is shown in Figure 5 (Tazawa et al., 2002). The distinct circadian rhythm of HR still remains, but the scope of diurnal maximum and nocturnal minimum dropped to about $100 \mathrm{bpm}$. The regression equation of MHR changes in three chicks after 30 days of post-hatch was:

$$
M H R=-|30| \cdot \cos (2 \pi x / 0.97)+330
$$

where $r=0.67$. The mean period of circadian rhythm is 0.97 day (about 23.3 hours). The parameters showing daily changes in HR; i.e. the scope of circadian rhythm and daily MHR, were both zero indicating that the circadian rhythm of chicken HR after 30 days becomes constant and independent of growth of the birds.

The circadian rhythm of HR is also absent during embryonic development in other domesticated birds; ducks and geese (Fukuoka et al., unpublished data) and emus (Kato et al., 2002). In hatchlings of these species, the circadian rhythm of HR were not distinct compared with chickens. In emus, some hatchlings showed

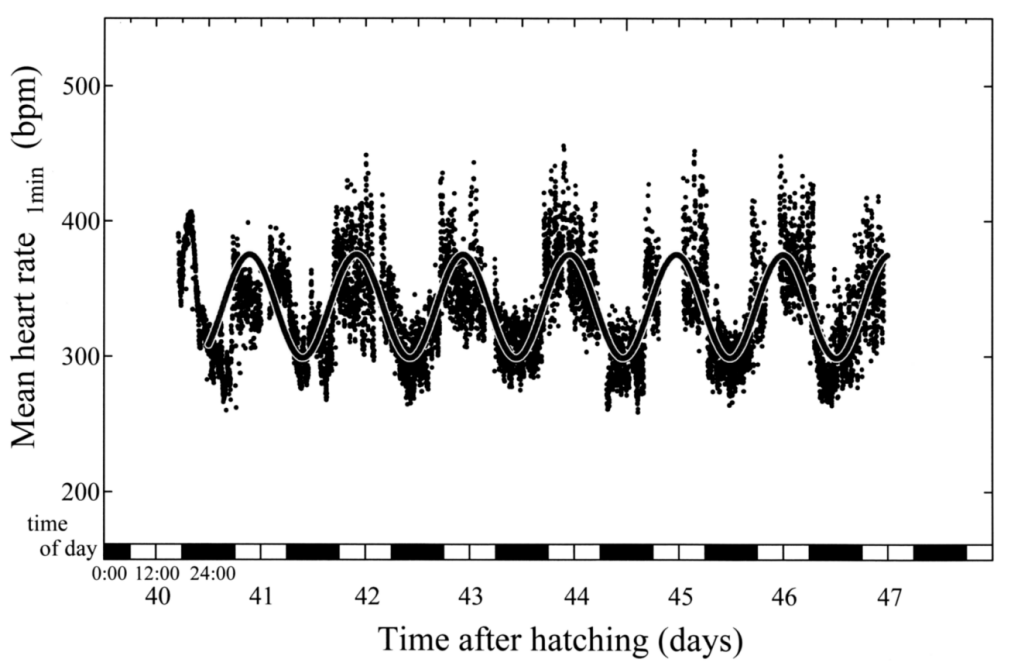

Fig. 5 The MHR recording and its regression function represented by $M H R=-|37| \cdot \cos (2 \pi x / 1.00)+337 \quad($ bpm $)$

$(r=0.75)$ in age from 40 to 47 days post hatch as adult chicken. Each dot indicates the MHR over a 1-minute period. This chicken was different from that illustrated in Figs 3 and 4. Modified from Tazawa et al. (2002). differences between the MHR of diurnal and nocturnal periods during the first few days of post-hatching life, but the scope was small, less than $100 \mathrm{bpm}$ (Moriya et al., 2002). In blue-naped mousebird, circadian rhythm of HR is reported, declining by $19.6 \%$ during the night (Schaub and Prinzinger, 1999).

The circadian pattern of HR change mirrors that of metabolic rate in adult birds (Berman and Meltzer, 1978). Metabolic rate has been shown to vary daily with a period of around 24-hours. The circadian rhythm of metabolic rate responded to the light-dark cycle. The two light-dark cycles within a day led to two cyclic periods of metabolic rate change.

\section{DEVELOPMENT OF CIRCADIAN RHYTHMS}

In mammals, the circadian rhythm of HR is mediated by the suprachiasmatic nucleus through the autonomic nervous system (Mutoh et al., 2003; Bujis et al., 2003). In the rat, exposure to light results in decreased vagal nerve activity, induces sympathoexcitation and increases HR (Mutoh et al., 2003). Bilateral lesions in the suprachiasmatic nucleus result in a disappearance of HR response to light. Further, the presence of melatonin inhibits the response to light. Increases in HR during the daytime may be due to decreases in the vagal tone.

It is likely that a similar pathway mediates the HR circadian rhythms in birds. The centres involved in controlling circadian rhythms in birds are thought to be the pineal gland, the suprachiasmatic nucleus and the eye (Yoshimura et al., 2001). The development of a circadian rhythm in chick HR occurs rapidly upon hatching and matures over the first week of post-hatch life (Figure 2). However, the mechanisms regulating circadian rhythms appear to be in place prior to hatching. Significant diurnal variation in the expression of the per 2 gene in both the pineal and suprachiasmatic nucleus occurs at about day 18 or 19 of incubation (Okabayashi et al., 2003) and continues to increase through hatching. Pineal gland and plasma melatonin concentrations in the embryo and hatchling follow a similar pattern with concentrations increasing during the first week of post- 
hatch life (Herichová et al., 2001). However, the rhythms in metabolism and HR do not develop until after hatching (Berman et al., 1978; Moriya et al., 2000; Tazawa et al., 2002). The developmental timing of the regulatory biological clocks follows closely the changes observed in the chick during the first week of post-hatch life.

It is possible that the increase in night and day HR during the first week of post-hatch life is due to further development of the autonomic nervous system. The autonomic nervous system is one of the main regulators of HR in the developing chicken (Crossley and Altimaris, 2000; Chiba et al., 2004). A

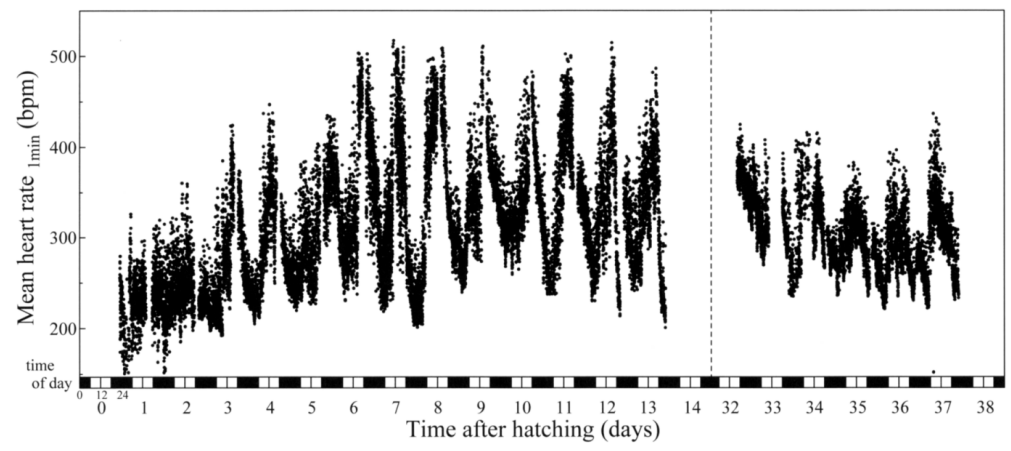

Fig. 6 The MHR recordings during days $0-14$ and days $32-38$ of post hatch. During the first and second weeks were measured from same individual (a different chicken from that illustrated in Figs 3 and 4), but it was different chicken from during days $32-38$. The recording during days $0-14$ is modified from Tazawa et al. (2002).

similar response of HR to warming and cooling has been observed in hatchlings during the first week of post-hatch life (Khandoker et al., 2004). Both cooling and warming increased HR during the first 3 to 4 days of post-hatch life followed by a plateau on days 5 to 7 . This change was attributed to maturation of the sympathetic nervous systems control of HR. Further, circadian rhythm in HR is most likely mediated by the autonomic nervous system. In the rat, HR changes during the night can be abolished by the administration of atropine (Makino et al., 1997). The development of a circadian rhythm and the increase in absolute HR is most likely due to maturation of both the sympathetic and parasympathetic nervous systems their interactions with the suprachiasmatic nucleus in a similar fashion as in mammals.

\section{CONCLUSIONS AND FUTURE DIRECTIONS}

\subsection{Conclusions}

A circadian rhythm is found visually to be absent in chick embryos until hatching. After hatching, the developmental pattern of MHR is determined from ECG measurement and shows distinct circadian rhythm of HR (Figure 6). The period of circadian rhythms is calculated to be approximately 24-hours. The daily MHR increases during the first week to almost constant level during the second week and in young chick. The amplitude of circadian rhythm also increases during the first week to the maximum scope during the second week. Then, the amplitude decreases in the young chick.

\subsection{Future directions}

It remains to be seen what factors control the development of the hatchling HR circadian rhythm. Though developmental pattern of HR changes occurred as hatching approached, distinct circadian rhythm of HR did not appear in late stages of chick embryo (Tazawa et al., 1999; Moriya et al., 2000). Potential regulators involved in circadian rhythms of HR and metabolic rate include hormone levels (e.g. melatonin, serotonin) related to dark-light cycle (Herichová et al., 2001; Zeman et al., 2001; Willbord et al., 2002; Haque et al., 2003) and development of the suprachiasmatic nucleus. Additionally, maturation of autonomic nervous function is an important regulatory factor of heart rate. The sympathetic nerve is thought to dominate during the day-time phase, with the parasympathetic nerve dominating the night-time period. The oscillating domination of the two main arms of the autonomic nervous system should be further examined.

Also of interest would be to see if the periodicity of cardiac rhythms can be affected by altering zeitgebers, and indeed whether the rhythms themselves can be suppressed or induced early in development by changing key environmental cues and whether this would affect the subsequent manifestation of cardiac rhythms in older animals.

In other avian species (i.e. ducks, geese and emus), the HR circadian rhythm is also absent during embryonic development and present in hatchlings. However, the HR circadian rhythm of their hatchlings is more unclear than chick hatchlings. Such clear circadian rhythm of HR may be distinctive of chickens or broiler breed used. A recent report suggests that 
different breeds of chickens may have different development of cholinergic chronotropic function (Crossley et al., 2003) and so investigation into development of HR circadian rhythm in other breeds remains to be made. The methodology and results reviewed in this paper suggest that the study of cardiac rhythms in avian development will be fertile ground for further study.

\section{ACKNOWLEDGEMENTS}

This research was supported in part by a Grant-in-Aid for Scientific Research awarded to K.M. (No. 14750355) of the Japan Society for Promotion of Science and by an NSF operating grant to WWB.

\section{REFERENCES}

Aschoff, J. and von Saint Paul, U. (1976) Brain temperature in the unanaesthetized chicken: its circadian rhythm of responsiveness to light. Brain Res., 101, 1-9.

Akiyama, R., Ono, H., Höchel, J., Pearson, J.T. and Tazawa, H. (1997) Noninvasive determination of instantaneous heart rate in developing avian embryos by means of acoustocardiogram. Med. Biol. Eng. Comput., 35, 323-327.

Akiyama, R., Matsuhisa, A., Pearson. J.T. and Tazawa, H. (1999). Long-term measurement of heart rate in chicken eggs. Comp. Biochem. Physiol., 124A, 469-474.

Berman, A. and Meltzer, A. (1978) Metabolic rate: its circadian rhythmicity in the female domestic fowl. J. Physiol., 282, 419-427.

Buijs, R.M., van Eden, C.G., Goncharuk, V.D. and Kalsbeek, A. (2003) The biological clock tunes the organs of the body: timing by hormones and the autonomic nervous system. $J$. Endocrinol., 177, 17-26.

Cain, J.R. and Abbott, U.K. (1970). A system for diurnal heart rate measurement in chickens. Poult. Sci., 49, 1085-1090.

Cain, J.R. and Wilson, W.O. (1974) The influence of specific environmental parameters on the circadian rhythms of chickens. Poult. Sci., 53, 1438-1447.

Chiba. Y., Fukuoka. S., Niiya. A., Akiyama. R. and Tazawa. H. (2004) Development of cholinergic chronotropic function in chick (Gallus gallus domesticus) embryos. Comp. Biochem. Physiol., in press.

Crossley, D. and Altimiras, J. (2000) Ontogeny of cholinergic and adrenergic cardiovascular regulation in the domestic chicken (Gallus gallus). Am. J. Physiol., 279, R1091-R1098.

Crossley, D.A., Hicks, J.W. and Thornburg, K. (2003) A comparison of cardiovascular regulatory mechanisms present during fowl ontogeny; white leghorn vs. bantam chickens. Society for Integrative and Comparative Biology, Abstract 147.

Haque, R., Alonso-Gomez, A.L., Chaurasia, S.S. and Invone, P.M. (2003) Diurnal regulation of arylalkylamine N-acetyltransferase activity in chicken retinal cells in vitro: analysis of culture conditions. Mol. Vis., 5, 52-59.
Herichová, I., Zeman, M., Macková, M. and Griač, P. (2001) Rhythms of the pineal N-acetyltransferase mRNA and melatonin concentrations during embryonic and post-embryonic development in chicken. Neurosci. Letters, 298, 123-126.

Katou, K., Moriya, K., Dzialowski, E., Burggren, W.W. and Tazawa, H. (2002) Cardiac rhythms in prenatal and perinatal emu embryos. Comp. Biochem. Physiol., 131A, 775-785.

Khandoker, A.H., Fukazawa, K., Dzialowski, E.M., Burggren, W.W. and Tazawa, H. (2004) Maturation of the homeothermic response of heart rate to altered ambient temperature in developing chick hatchlings (Gallus gallus domesticus). Am. J. Physiol., 286, 129-138.

Makino, M., Hayashi, H., Takezawa, H., Hirai, M., Saito, H. and Ebihara, S. (1997) Circadian rhythms of cardiovascular functions are modulated by the baroreflex and the autonomic nervous system in the rat. Circulation, 96, 1667-1674.

Moriya, K., Höchel, J., Pearson, J.T. and Tazawa, H. (1999) Cardiac rhythms in developing chicks. Comp. Biochem. Physiol., 124A, 463-470.

Moriya, K., Kato, K., Matsumura, M., Dzialowski, E.M., Burggren, W.W. and Tazawa, H. (2002) Cardiac rhythms in developing emu hatchlings. Comp. Biochem. Physiol., 131A, 787-795.

Moriya, K., Pearson, J.T., Burggren, W.W., Ar, A. and Tazawa, H. (2000) Continuous measurement of instantaneous heart rate and it's fluctuations before and after hatching in chickens. $J$. Exp. Biol., 203, 895-903.

Mutoh, T., Shibata, S., Korf, H.W. and Okamura, H. (2003) Melatonin modulates the light-induced sympathoexcitation and vagal suppression with participation of the suprachiasmatic nucleus in mice. J. Physiol., 547, 317-332.

Nichelmann, M., Höchel, J. and Tzschentke B. (1999) Biological rhythms in birds - development, insights and perspectives. Comp. Biochem. Physiol., 124A, 429-437.

Okabayashi, N., Yasuo, S., Watanabe, M., Namikawa, T., Ebihara, S. and Yoshimura, T. (2003) Ontogeny of circadian clock gene expression in the pineal and the suprachiasmatic nucleus of chick embryo. Brain Res., 990, 231-234.

Rahn, H., Poturalski, S.A. and Paganelli, C.V. (1990) The acoustocardiogram: a noninvasive method for measuring heart rate of avian embryos in ovo. J. Appl. Physiol., 69, 1546-1548.

Schaub, R. and Prinzinger, R. (1999) Long-term telemetry of heart rates and energy metabolic rate during the diurnal cycle in normothermic and torpid African blue-naped mousbirds (Urocolius macrourus). Comp. Biochem. Physiol., 124A, 439-445.

Shimada, K. and Koide, H. (1978) Effects of age, light regimes and food removal on development of daily rhythmicity in chicken heart rate. Poult. Sci., 57, 271-276.

Tazawa, H., Akiyama, R. and Moriya, K. (2002) Development of cardiac rhythms in birds. Comp. Biochem. Physiol., 132A, $675-689$.

Tazawa, H., Mitsubayashi, H., Hirata, M., Höchel, J. and Pearson, J.T. (1999) Cardiac rhythms in chick embryos during hatching. Comp. Biochem. Physiol., 124A, 511-521.

Tazawa, H., Takami, M., Kobayashi, K., Hasegawa, J. and Ar, A. (1992) Non-invasive determination of heart rate in newly hatched chicks. Br. Poult. Sci., 33, 1111-1118.

Wang, N., Butler, J.P. and Banzett, R.B. (1990) Gas exchange across avian eggshells oscillates in phase with heartbeat. $J$. Appl. Physiol., 69, 1546-1552. 
Willbord, E., Huhn, J., Korf, H.W., Voisin, P. and Layer P.G. (2002) Light-dark and circadian rhythms are established de novo in re-aggregates of the embryonic chicken retina. Dev. Neurosci., 24, 504-511.

Yoshimura, T., Yasuo, S., Suzuki, Y., Makino, E., Yokota, Y. and Ebihara, S. (2001) Identification of the suprachiasmatic nucleus in birds. Am. J. Physiol., 280, R1185-R1189.
Zeman, M., Buyse, J., Herichová, I. and Decuypere, E. (2001) Melatonin decreases heat production in female broiler chickens. Acta. Vet. Brono, 70, 15-18.

Zeman, M., Gwinner, E., Herichova, I. Lamosova, D., Kostál, L. (1999) Perinatal development of circadian melatonin production in domestic chicken. J. Pineal. Res., 26, 28-34. 